\title{
Bulk Nanostructured Metals and Alloys: Processing, Structure, and Thermal Stability
}

\author{
Hamed Bahmanpour, ${ }^{1}$ Amir Kajbafvala, ${ }^{2}$ Mohammad H. Maneshian, ${ }^{3}$ \\ Hamid Reza Zargar, ${ }^{4}$ and Khaled Youssef ${ }^{2}$ \\ ${ }^{1}$ Department of Chemical Engineering and Materials Science, University of California, Davis, CA 95616, USA \\ ${ }^{2}$ Department of Materials Science and Engineering, North Carolina State University, Raleigh, NC 27695-7907, USA \\ ${ }^{3}$ Department of Materials Science and Engineering, University of North Texas, Denton, TX 76203, USA \\ ${ }^{4}$ Department of Metals and Materials Engineering, University of British Columbia, Vancouver, BC, Canada V6T 1Z4
}

Correspondence should be addressed to Hamed Bahmanpour, hbahman@ncsu.edu

Received 21 October 2012; Accepted 21 October 2012

Copyright (๑) 2012 Hamed Bahmanpour et al. This is an open access article distributed under the Creative Commons Attribution License, which permits unrestricted use, distribution, and reproduction in any medium, provided the original work is properly cited.

Nanostructured metals and alloys have been the focus of research during the past few decades due to their interesting microstructures and promising mechanical properties. Despite the fact that this class of materials shows intriguing properties, there are roadblocks that prevent large-scale production and industrial application of these materials that include but not limited to low ductility and poor thermal stability. In this regard, tailoring mechanical and microstructural properties by developing new alloys and inducing different microstructural features via various processing routes have been the major point of interest for researchers to overcome the difficulties in industrialization of nanomaterials. High tendency of nanograins to reduce their energy by grain coalescence and growth brings the thermodynamics and kinetics of grain growth to attention in order to understand the phenomena and develop thermally stable nanostructures. Attempts in this regard include reducing the mobility of a grain boundary by means of mechanisms such as solute drag, second-phase particle pinning, and chemical ordering. On the other hand, the demand for producing large-sized bulk parts, especially in structural applications, has led to the emergence of new processing techniques and further application-based approaches to the issue of the nanostructured metals and alloys. This special issue addresses the different challenges for up scaling the production of nanostructured metals and alloys with emphasis on processing, microstructure, and thermal stability. We would like to express our appreciation to all authors in this special issue for their help and efforts in addressing these current issues in the field. Furthermore, thanks are extended to all reviewers for enhancing the quality of these papers.

This special issue contains six papers related to synthesis and characterization of nanostructured metals and alloys. In "Thermal stability of neodymium aluminates high-k dielectric deposited by liquid injection MOCVD using single-source heterometallic alkoxide precursors," P. Taechakumput et al. studied the effects of high-temperature post deposition annealing (PDA) on the properties of the $\mathrm{NdAlO}_{x}$ thin films, deposited by metalorganic chemical vapor deposition (MOCVD) using single-source precursor. These $\mathrm{NdAlO}_{x}$ thin films were shown to remain amorphous up to $50^{\circ} \mathrm{C}$ as indicated by XRD. No significant level of crystallinity or movement of metal ions was evident after annealing at $950^{\circ} \mathrm{C}$ as indicated by MEIS energy spectra. Good electrical integrity was maintained even after $950^{\circ} \mathrm{C}$ PDA showing the extracted dielectric permittivity of 12 , a low leakage density of $7 \times 10^{-7} \mathrm{Acm}^{-2}$ at $2 \mathrm{MV} \mathrm{cm}^{-1}$, and a density of interface states at flat band $D_{\text {it }}$ of $4.01 \times 10^{11} \mathrm{~cm}^{-2} \mathrm{eV}^{-1}$. These features make the neodymium aluminate a potential candidate for the dielectric replacement.

In their paper, Z. S. Hu et al. studied "Align Ag nanorods via oxidation reduction growth using RF-sputtering" and demonstrated an oxidation reduction growth (ORG) technique with mixed-gas sputtering to create Ag nanorod arrays via oxide-assisted growth without any chemical solutions or contamination from aqueous solution. The ORG methodology is used to deposit an Ag buffer layer with 
silver oxide nanoclusters to obtain Ag nanorod arrays using a two-step mixed gaseous process. The Ag nanorods grew in the original locations of reduced metal nuclei after thermal composition of silver oxide nanoclusters. The Ag nanorods stood vertically on the Ag buffer interlayer and grew from the interface between the Ag grains and the Ag buffer interlayer. The success of the technique provides support for the oxidation reduction growth (ORG) mechanism and proves suitable for fabrication of $\mathrm{Ag}$ nanorods in the semiconductor industry. Due to photoactivation caused by radiative recombination of Fermi level electrons and $\mathrm{d}$ band holes, the observed photoluminescence spectra of the $\mathrm{Ag}$ nanorods were observed to be $2.17 \mathrm{eV}$ in both air and vacuum.

In "Synthesis of bulk nanostructured $\mathrm{DO}_{22}$ superlattice of $\mathrm{Ni}_{3}(\mathrm{Mo}, \mathrm{Nb})$ with high strength, high ductility, and high thermal stability," H. M. Tawancy showed that bulk nanostructured $\mathrm{DO}_{22}$-type superlattice with high strength, high ductility, and high thermal stability can be synthesized in a Ni-Mo-Nb alloy with a composition approaching $\mathrm{Ni}_{3}(\mathrm{Mo}, \mathrm{Nb})$ by a simple aging heat treatment at $700^{\circ} \mathrm{C}$. Upon thermal aging, the grains of the high temperature fcc phase are subdivided into ordered crystals on the nanoscale (10-20 nm) with room-temperature yield strength of about $820 \mathrm{MPa}$ and tensile ductility of $35 \%$. Plastic deformation in the ordered state is found to predominantly occur by twinning on (111) planes of the parent fcc structure indicating that the superlattice preserves the twinning systems of the parent phase leading to the observed high ductility.

In their paper, E. Huerta et al. presented "Elastic modulus determination of $\mathrm{Al}-\mathrm{Cu}$ film alloys prepared by thermal diffusion." In this study, the elastic modulus of Al-50 at.\% $\mathrm{Cu}$ alloy films with $50-250 \mathrm{~nm}$ thickness, prepared by thermal evaporation on Kapton $50 \mathrm{HN}$ flexible substrates and postthermal diffusion, was investigated. The morphology and mechanical properties of the Kapton foil substrate and metallic alloys were investigated to distinguish features and properties of the formed Al-Cu alloy from the bare substrate. Pure $\mathrm{Al}$ and $\mathrm{Cu}$ films with thickness of $50 \mathrm{~nm}$ were analyzed for comparison. The elastic modulus of 50-250 nm thick Al-50 at.\% Cu alloys thermally evaporated on to Kapton substrates and postformed by thermal diffusion was investigated. $\mathrm{Al}_{2} \mathrm{Cu}$ phase was the dominant crystalline phase formed as determined by XPS. Force-strain curves of the $\mathrm{Al}-\mathrm{Cu}$ alloys were obtained by subtracting the force-strain curve of the Kapton substrate from the force-strain curve of the Al-Cu/Kapton material system. The elastic modulus was obtained from the slope of the corresponding stress-strain curves. Elastic modulus of the Al-Cu alloys decreased as the film thickness increased, and their values were determined to be in the range of 106.1 to $77.8 \mathrm{GPa}$ for 50 to $250 \mathrm{~nm}$ thick alloys, respectively. The elastic modulus of the studied Al-Cu alloys was found to lie between the corresponding bulk values of the $\mathrm{Al}$ and $\mathrm{Cu}$ films. The elastic modulus measured for $50 \mathrm{~nm}$ thick Al was higher than its corresponding bulk value, while the elastic modulus of $50 \mathrm{~nm}$ thick $\mathrm{Cu}$ was smaller than its bulk value. The highest values of the mean grain size and rms-roughness were found for the $150 \mathrm{~nm}$ thick Al-Cu alloy. The methodology used to obtain the elastic modulus does not yield alloy microfractures because of the small strains $(<1 \%)$ applied during tensile testing.

In their paper, M. Tavoosi and coworkers studied "Consolidation of amorphous $\mathrm{Al}_{80} \mathrm{Fe}_{10} \mathrm{Ti}_{5} \mathrm{Ni}_{5}$ powders by hot pressing." The purpose of their study was to investigate the feasibility of fabricating amorphous $\mathrm{Al}_{80} \mathrm{Fe}_{10} \mathrm{Ti}_{5} \mathrm{Ni}_{5}$ powders by mechanical alloying and consolidation into bulk samples by a hot-pressing technique. The crystallization process of this amorphous alloy is a one-stage mode of the $\mathrm{Al}_{13}(\mathrm{Fe}, \mathrm{Ti})_{4}$ and $\mathrm{Al}_{3} \mathrm{Ti}$ intermetallic compounds. The results showed that the as-milled amorphous $\mathrm{Al}_{80} \mathrm{Fe}_{10} \mathrm{Ti}_{5} \mathrm{Ni}_{5}$ powders were consolidated successfully into bulk metallic glasses by a hot-pressing technique. The temperature and pressure for successful condensation of amorphous powders in the hotpressing method were $550^{\circ} \mathrm{C}$ and $600 \mathrm{MPa}$, respectively. During the consolidation, the amorphous phase does not remain, and an AlTi intermetallic phase precipitates in the amorphous matrix.

In their paper, K. S. Lin et al. investigated "Synthesis and characterization of metal hydride/carbon aerogel composites for hydrogen storage." The synthesis, characterization, and $\mathrm{H}_{2}$ adsorption capacity of carbon aerogels (CAs)/metallic hydride nanocomposites as a catalyst were studied. Experimentally, The $\mathrm{H}_{2}$ storage capacity of metallic samples was measured by a TGA microbalance method. In addition, fine structure and crystallinity of metallic hydride was identified by BET nitrogen adsorption isotherms, HRTEM, FE-SEM/EDS, XRD, and XANES/EXAFS. The carbon aerogels were very effective in improving the hydrogen storage capacity of the Fe-, Ti-doped $\mathrm{MgH}_{2}$ samples with the "hydrogen spillover" route. Additionally, metallic dispersion of $\mathrm{Pd}$ nanoparticles onto the CAs might improve the hydrogen adsorption abilities. Higher surface area from CAs and the defect criteria of the $\mathrm{MgH}_{2}$ surface due to the doping of the metallic particle cause improvement in the hydrogen adsorption capacities. Conversely, Ti-doping onto the sodium aluminum hydride only improves slightly the hydrogen storage capabilities.

\author{
Hamed Bahmanpour \\ Amir Kajbafvala \\ Mohammad H. Maneshian \\ Hamid Reza Zargar \\ Khaled Youssef
}



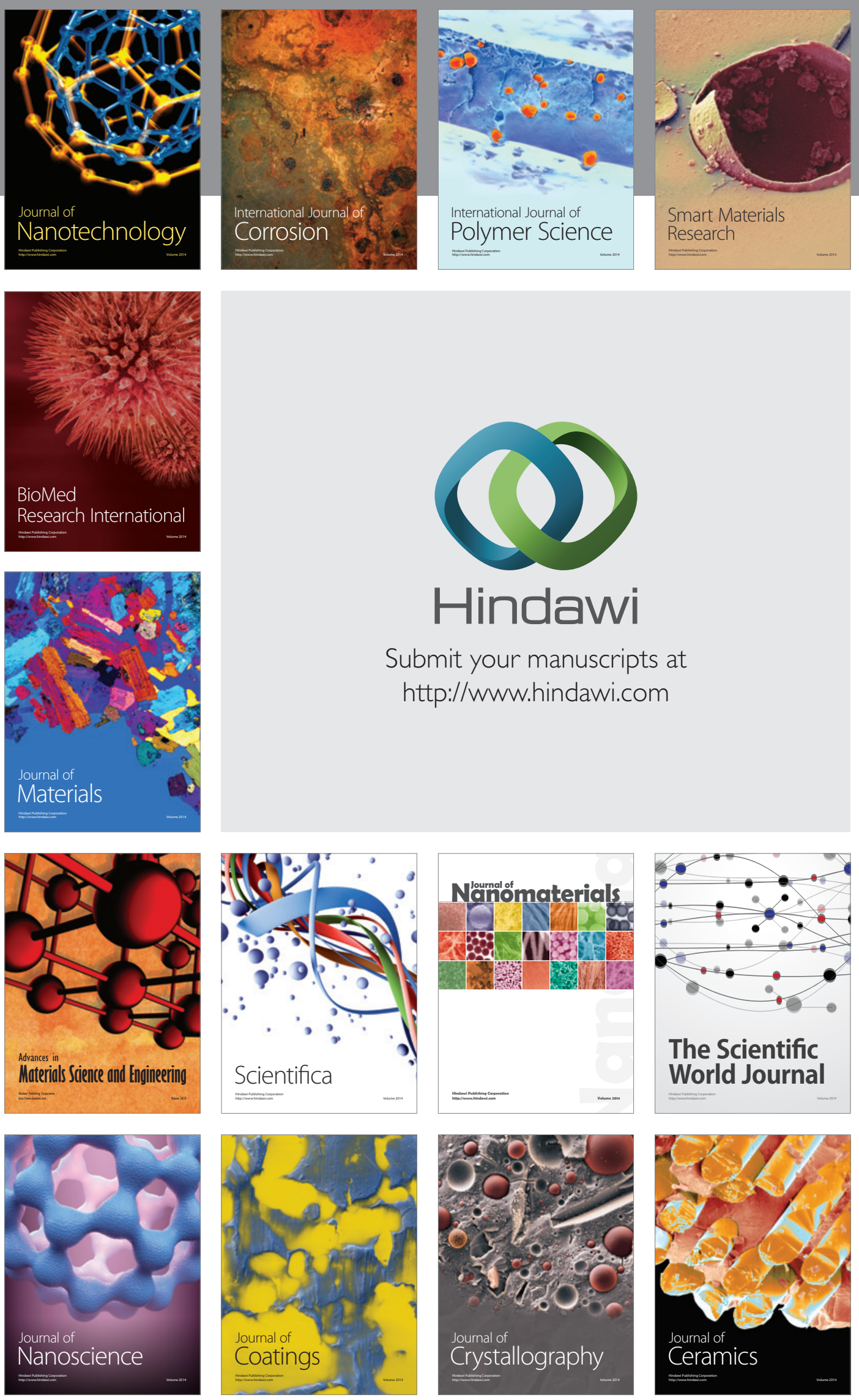

The Scientific World Journal

Submit your manuscripts at

http://www.hindawi.com

\section{World Journal}

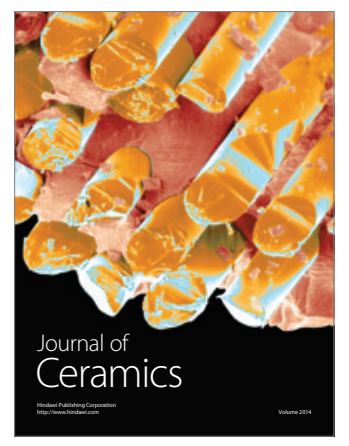

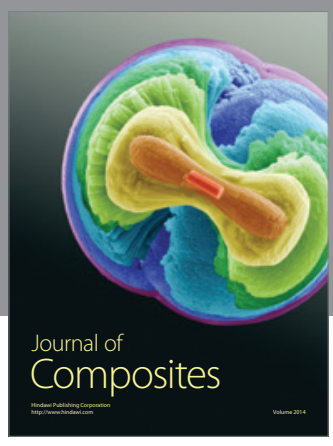
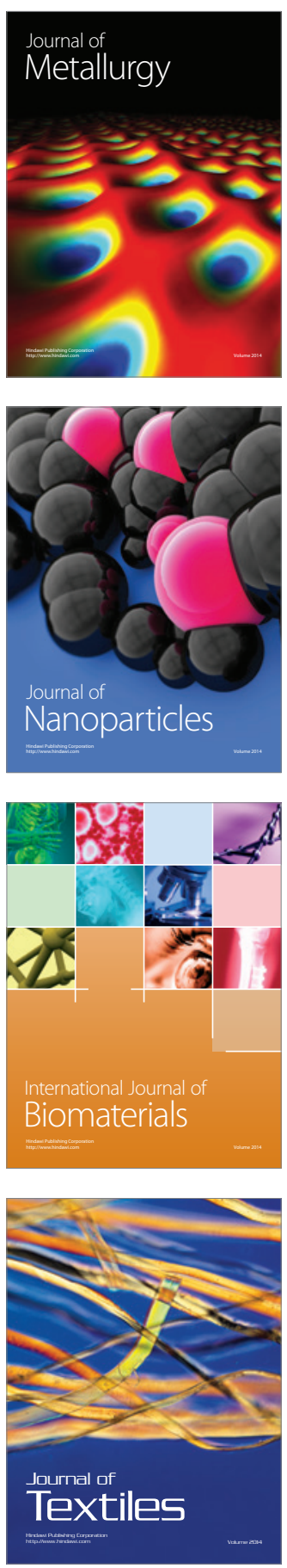